\title{
Molecular simulation of interfacial reaction between TiAl alloy melts and different coatings
}

\section{Yan-wei Sui, Cheng Cheng, Kun Feng, *Ji-qiu Qi, Ye-zeng He, Fu-xiang Wei, Qing-kun Meng and Zhi Sun} School of Material Science and Engineering, China University of Mining and Technology, Xuzhou 221116, China

\begin{abstract}
The effect of coatings $\left(\mathrm{Y}_{2} \mathrm{O}_{3}, \mathrm{ZrO}_{2}\right.$ and $\left.\mathrm{Al}_{2} \mathrm{O}_{3}\right)$ on the interfacial reaction of TiAl alloys was studied with molecular dynamics. The binding energy of coatings and the diffusion process of oxygen in the melt were simulated, and then the simulation results were compared with the experimental results. The simulation results indicate that for each of the three simulated coatings, inordinate interfacial reactions have occurred between the coating and the melt. The binding energy results show that $\mathrm{Y}_{2} \mathrm{O}_{3}$ has the best stability and is the most difficult to break down. $\mathrm{ZrO}_{2}$ has the greatest decomposition energy and is the easiest to break down in the melt. Besides, the molecular dynamics indicate that the diffusion coefficient of the oxygen atom in $\mathrm{Al}_{2} \mathrm{O}_{3}$ is larger than that in the other two coatings, indicating that oxygen diffusion in $\mathrm{Al}_{2} \mathrm{O}_{3}$ is the fastest at a given temperature. The experimental results show that the oxygen concentration of the melt with $\mathrm{Al}_{2} \mathrm{O}_{3}$ coating is the highest, and the oxygen diffusion is of similar magnitude to the simulation values, from which the conclusion can be obtained that the oxygen concentration is significantly influenced by the coating materials.
\end{abstract}

Key words: TiAl alloy; interfacial reaction; molecular simulation; coating material
CLC numbers: TG146.23
Document code: $\mathrm{A}$
Article ID: 1672-6421(2017)03-205-06

$\mathrm{T}$ iAl alloys exhibit many excellent properties such as low density, high melting temperature, good elevatedtemperature strength, Young's modulus, high resistance to oxidation, and excellent creep properties ${ }^{[1-2]}$. Thus, TiAl alloys are extensively applied in the gas turbine and automotive industries ${ }^{[3-4]}$. However, the poor ductility at room temperature hinders the development of TiAl alloys ${ }^{[5]}$. Some research results showed that TiAl alloys with fully lamellar structure, which could be obtained by controlling the crystallographic orientation using the directional solidification technique ${ }^{[6]}$, have a well-balanced combination of strength, ductility and other properties. Nevertheless, during the directional solidification of TiAl alloys, the high reactivity between the molten alloy and the ceramic crucible materials such as graphite and alumina brings in defects and contamination, which contributes to the poor mechanical properties ${ }^{[7]}$.

Zhang et al. ${ }^{[8-9]}$ used $\mathrm{Y}_{2} \mathrm{O}_{3}$ coated $\mathrm{Al}_{2} \mathrm{O}_{3}$ crucibles to produce TiAl alloys with directional solidification, and found that the surface of the castings had been effectively protected. These double layer crucibles are

\section{*Ji-qiu Qi}

Male, born in 1983, Ph.D. Research interests: preparation of metal matrix composites and analysis of their microstructure and properties.

E-mail: qijiqiu_hit@163.com

Received: 2016-08-21; Accepted: 2017-01-26 used in the directional solidification of TiAl alloys owing to a reasonable cost and relatively high service performance. Recently, $\mathrm{Y}_{2} \mathrm{O}_{3}$ and $\mathrm{ZrO}_{2}$ have been used as coatings in directional solidification to produce TiAl alloys ${ }^{[10-12]}$. But, so far, no crucible materials have been found to be absolutely inert to TiAl melts, and some interactions between the crucibles and the molten metal always occur during melting and casting ${ }^{[13-14]}$.

Molecular modeling techniques have been widely applied to materials as a way to better understand the structure of materials and their interactions on the atomic scale. Simulation-derived diffusion rates can be used to evaluate the interfacial reaction processes. Molecular dynamics (MD) simulations may be adopted to calculate the diffusion coefficient for a variety of structures more accurately, and the diffusion coefficients of oxygen and oxide molecules in simulated systems were verified by many researchers ${ }^{[15]}$.

The reaction between TiAl alloys and coatings are accompanied by the diffusion of oxygen atoms and the change of energy ${ }^{[16]}$. This research was focused on the modeling of the oxygen diffusion. In addition, there are no relevant reports on computational energy which applied molecular dynamics methods to the interaction. Thus, the diffusion process of oxygen and the binding energy of coatings are studied through molecular dynamics simulations, so as to provide a new understanding of the 
interaction between TiAl alloys and coatings at the molecular level. Finally, the experiment values of the oxygen diffusion are compared with the simulation results.

\section{Simulation}

The Ti-48Al model whose lattice parameters are $\mathrm{a}=\mathrm{b}=4.10 \AA$, $c=4.18 \AA ; \alpha=\beta=\gamma=90^{\circ}{ }^{[17]}$ was built with the Amorphous Cell module of the Materials Studio. Then, after its energy was optimized (see Fig.1), this model was piled into the amorphous units with the specified density and periodic boundary conditions. The COMPASS (Condensed-phase Optimized Molecular Potentials for Atomistic Simulation Studies) forcefield was used for modeling all three Para and Meta isomer pairs in an atomistic way. The bonded parameters for pairs of different atoms are calculated by the software automatically to match program via combining rules. Subsequently, the model was subjected to an equilibration process using an appropriate ensemble which can be seen as the integration of many relatively independent systems with the same structure and the same property under various motion constraint conditions. Finally, the Ti-48Al model was thermally equilibrated using an initial 50 picosecond (ps) simulation in the microcanonical ensemble (particle number $\mathrm{N}$, volume $\mathrm{V}$, thermodynamic temperature T).

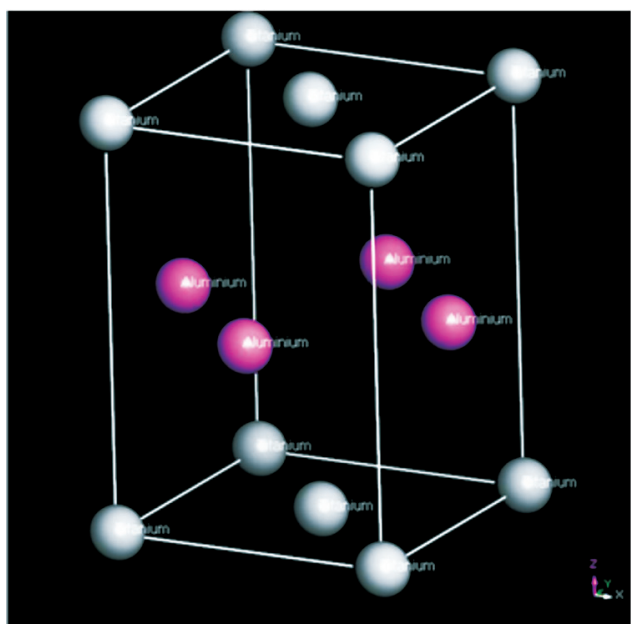

Fig. 1: Ti-48Al crystal model

Prior to the simulations, the model was geometrically optimized through an iterative process until the potential energy and forces converged to a constant value and achieved the minimum energy arrangement of atoms in the crystal. With this specified set of potential values, all the atoms were able to retain their structural symmetry. Then, an optimized structure with the minimized energy was obtained as shown in Fig. 2.

After the geometrical optimization, a supercell containing $10 \times 10 \times 4$ repeats in the $\mathrm{x}, \mathrm{y}$, and $\mathrm{z}$ directions was created, with approximate dimensions in the $x, y$ plane of $410 \AA \times 410 \AA$. Supercell volume changes were allowed in the $\mathrm{z}$ direction only $(\mathrm{x}$ and $y$ remained fixed at their optimized values). The composite vacuum box of TiAl alloys supercell is shown in Fig. 3 .

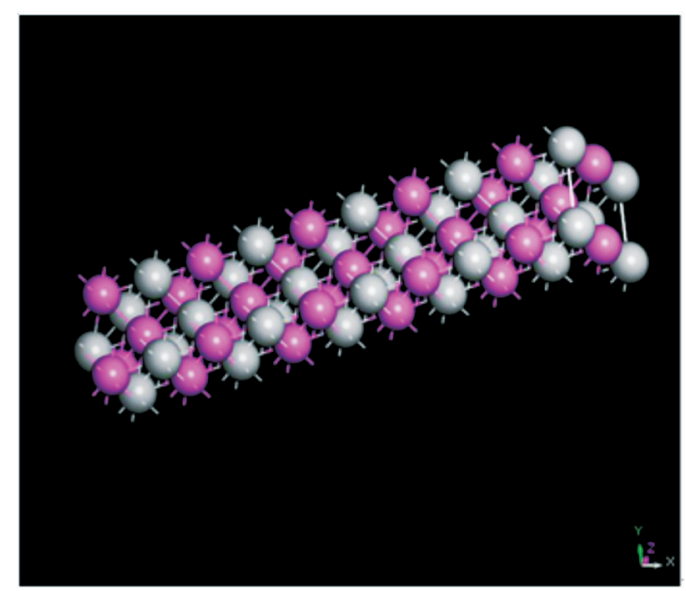

Fig. 2: Surface model

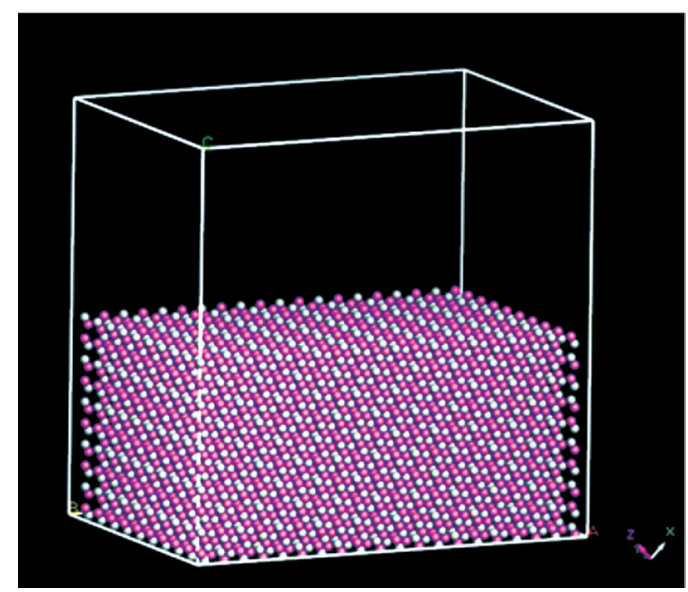

Fig. 3: Composite vacuum box of TiAl alloys supercell

$\mathrm{Y}_{2} \mathrm{O}_{3}, \mathrm{ZrO}_{2}$ and $\mathrm{Al}_{2} \mathrm{O}_{3}$ were chosen as the coating materials. Their molecular models were added to the TiAl supercell model at a distance of $30 \AA$. MD simulations were performed using the Forcite module of Material Studio. The time step in short-range interactions was evaluated per $1 \mathrm{fs}$ with a real-space cut-off radius of $9.5 \AA$. Interactions between molecules were described by means of long range Coulomb interactions calculated by Ewald summation. Fifty initial states were constructed for each model and followed by 5,000 steps of energy minimization at 1,873 K. Afterwards, a 60 ps MD run was performed for per 50 states to equilibrate in NVT ensemble.

In the calculating phase (NVT-MD simulation), there is a standard atom space-time correlation fluctuation that can be taken to judge whether an MD simulation is balanced. In general, if the fluctuation of the atom space-time correlation lies between $0.5 \%$ and $1 \%$, the system is considered to reach equilibrium. To obtain the precision analyzed results, all the three calculations of the atom space-time correlation are to a stable value after $200 \mathrm{ps}$. It means that the MD simulations were balanced under the condition of system equilibrium. The atom space-time correlation profiles of the Ti-Al melt/oxides blend system are shown in Fig. 4. 

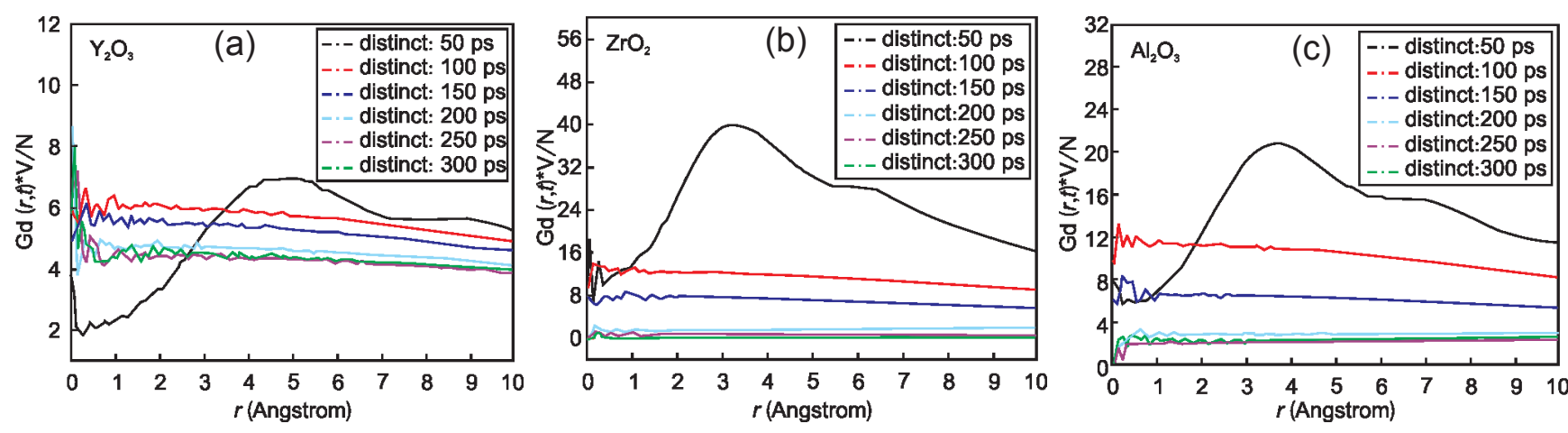

Fig. 4: Atom space-time correlation function for different coatings

\section{Results and discussion}

\subsection{Binding energy simulation of interfacial reaction}

In order to investigate the effects of the coating materials on the extent of interfacial reaction, several interfacial molecules $\left(\mathrm{Y}_{2} \mathrm{O}_{3}\right.$, $\mathrm{Al}_{2} \mathrm{O}_{3}$ and $\mathrm{ZrO}_{2}$ ) were added into the Ti-48Al model constructed above. And all the simulation steps were the same as the previous progress. Binding energy can well reflect the capacity of components blending with each other. It was defined as the intermolecular interaction energy when its negative value was taken, and the greater the value, the more stable the blend system. Binding energy was expressed as follows in Equation (1) ${ }^{[18-19]}$.

$$
E_{\text {interaction }}=E_{\text {total }}-\left(E_{\mathrm{TiAl}}+E_{\text {oxide }}\right)
$$

where $E_{\text {total }}$ is the total energy of TiAl alloy melt/oxides blend after MD calculation, $E_{\mathrm{TiAl}}$ is the point energy of TiAl alloy before calculation, and $E_{\text {oxide }}$ is the point energy of different oxides before calculation.

In order to compare the interaction strength between different compositions, three models with distinct contrast were excerpted to calculate the binding energy. In the models with different coating materials, i.e., $\mathrm{Y}_{2} \mathrm{O}_{3}$ with a binding energy of $560 \mathrm{kcal} \cdot \mathrm{mol}^{-1} ; \mathrm{Al}_{2} \mathrm{O}_{3}$ with a binding energy of $800 \mathrm{kcal}^{\circ} \mathrm{mol}^{-1}$ and $\mathrm{ZrO}_{2}$ with a binding energy of $900 \mathrm{kcal} \cdot \mathrm{mol}^{-1}$. The binding energies (negative) calculated by MD simulation are shown in Fig. 5.

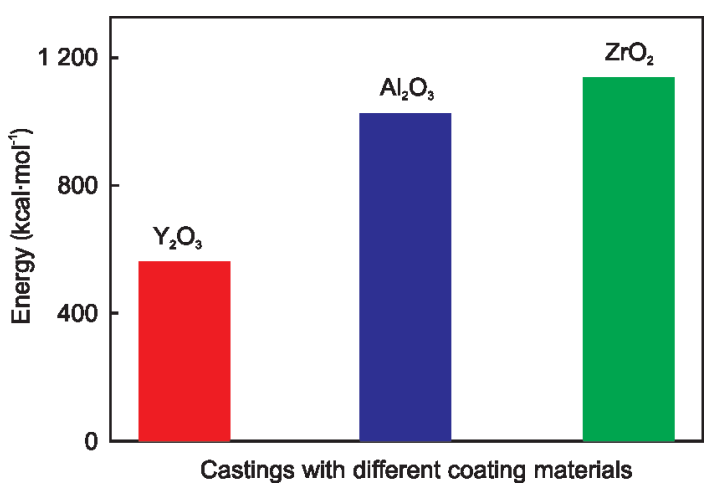

Fig. 5: Binding energy of different coating oxides with TiAl alloy melt
The binding energies of three blend systems showed that $E_{\text {total }}$ is different for different coating materials. That is, different coating materials led to different interaction forces among molecular chains. $\mathrm{ZrO}_{2}$ coating system had the largest binding energy value which is why the system had the strongest interaction. Moreover, by comparing the single point energies of different coatings, it was easy to find that the single point energy of $\mathrm{Y}_{2} \mathrm{O}_{3}$ was lower than that of the other two coating materials; this result was consistent with binding energy. Therefore, a stronger intermolecular interaction existed in the $\mathrm{ZrO}_{2}$ coating system compared with other coating materials of the same quality. The strength of the intermolecular interaction had the order of $\mathrm{ZrO}_{2}>\mathrm{Al}_{2} \mathrm{O}_{3}>\mathrm{Y}_{2} \mathrm{O}_{3}$.

After MD simulation, the interfacial reaction states of the interaction systems with different coatings at $1,873 \mathrm{~K}$ were obtained, as shown in Fig. 6. Obviously, the interfacial reaction of different coatings all reacted within the supercell, which can also be known from the network of the blend system.

\subsection{Calculation of oxygen diffusion coefficients}

Simulations for oxygen diffusion were carried out on the supercell box under periodic boundary conditions. The structure of the supercell was based on the same parameter as molecular dynamics. Atomic coordinates from the final 300 ps of NPT (particle number $\mathrm{N}$, pressure $\mathrm{P}$, thermodynamic temperature $\mathrm{T}$ ) ensemble simulation per $1.0 \mathrm{fs}$ were stored and were used for diffusion analysis.

In the actual calculation, the curve of the mean square displacement (MSD) with time (logarithm) can be drawn to check the reliability of the results ${ }^{[20]}$. If the calculation results were converged, a category straight line can be achieved. Plots of MSD (logarithm) versus time from simulations showed that the linear behavior changed over time, and this problem had been minimized by restarting the calculation at each data frame (2.0 fs). Diffusion data of oxygen ions can be collected from MSD curves obtained from the simulation run of 100 ps using a NVT ensemble with the Hoover Thermostat. The log (MSD) convergence curve for 300 ps simulation is shown in Fig. 7. The alumina coating had the best corresponding linear convergence 

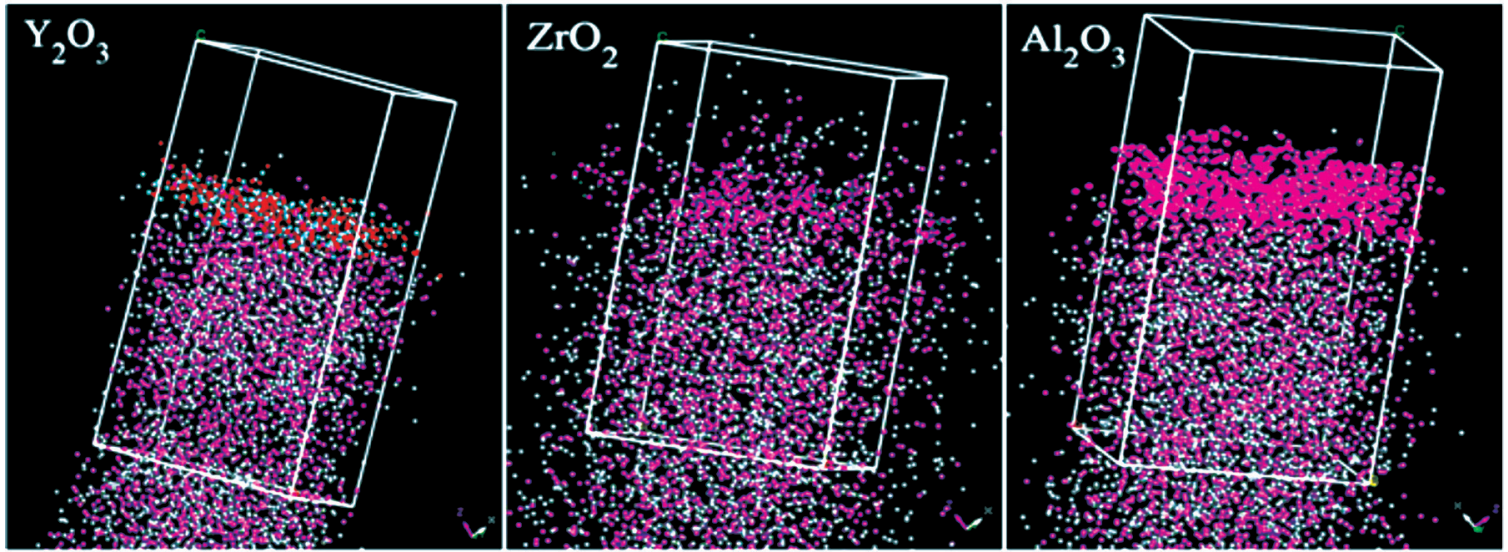

Fig. 6: Interfacial reaction states of different coatings at equilibrium system
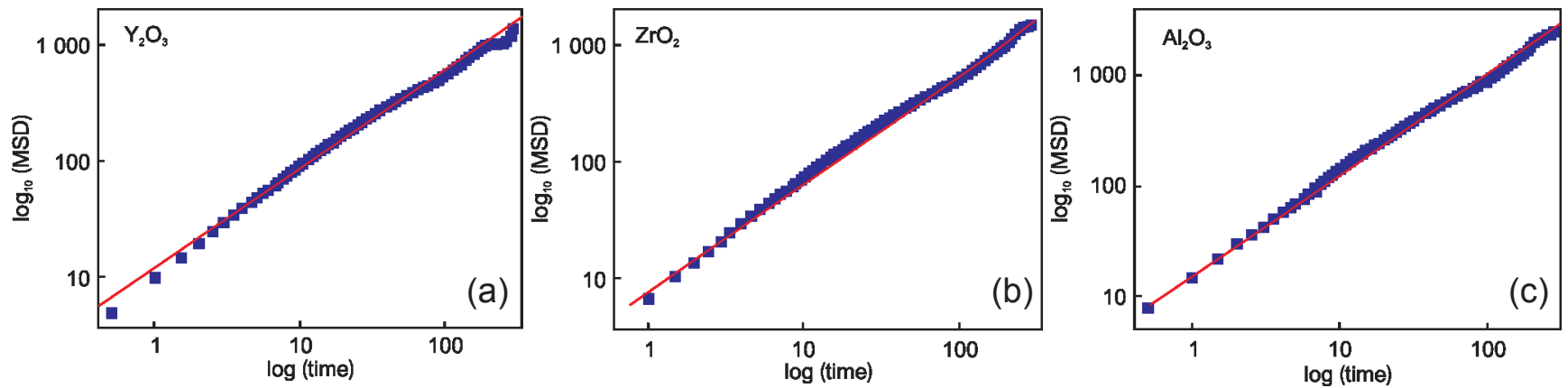

Fig. 7: MSD convergence figures for different coatings

diagram, which reflected that the diffusion characteristic of alumina coating is the best in TiAl alloy.

The curves in Fig. 7 indicate that the MSD convergence of different coatings will tend to be linear after the number of repeat units exceeds 30 . So the plots of MSD versus time are linear for all coatings over a period time of about $100 \mathrm{ps,} \mathrm{and}$ all estimated diffusion coefficients $(D)$ can be obtained from the linear parts of the plots. The MSD data were gathered and converted into the diagram as shown in Fig. 8. The block region in the figures reflected the stability degree of oxygen MSD, and
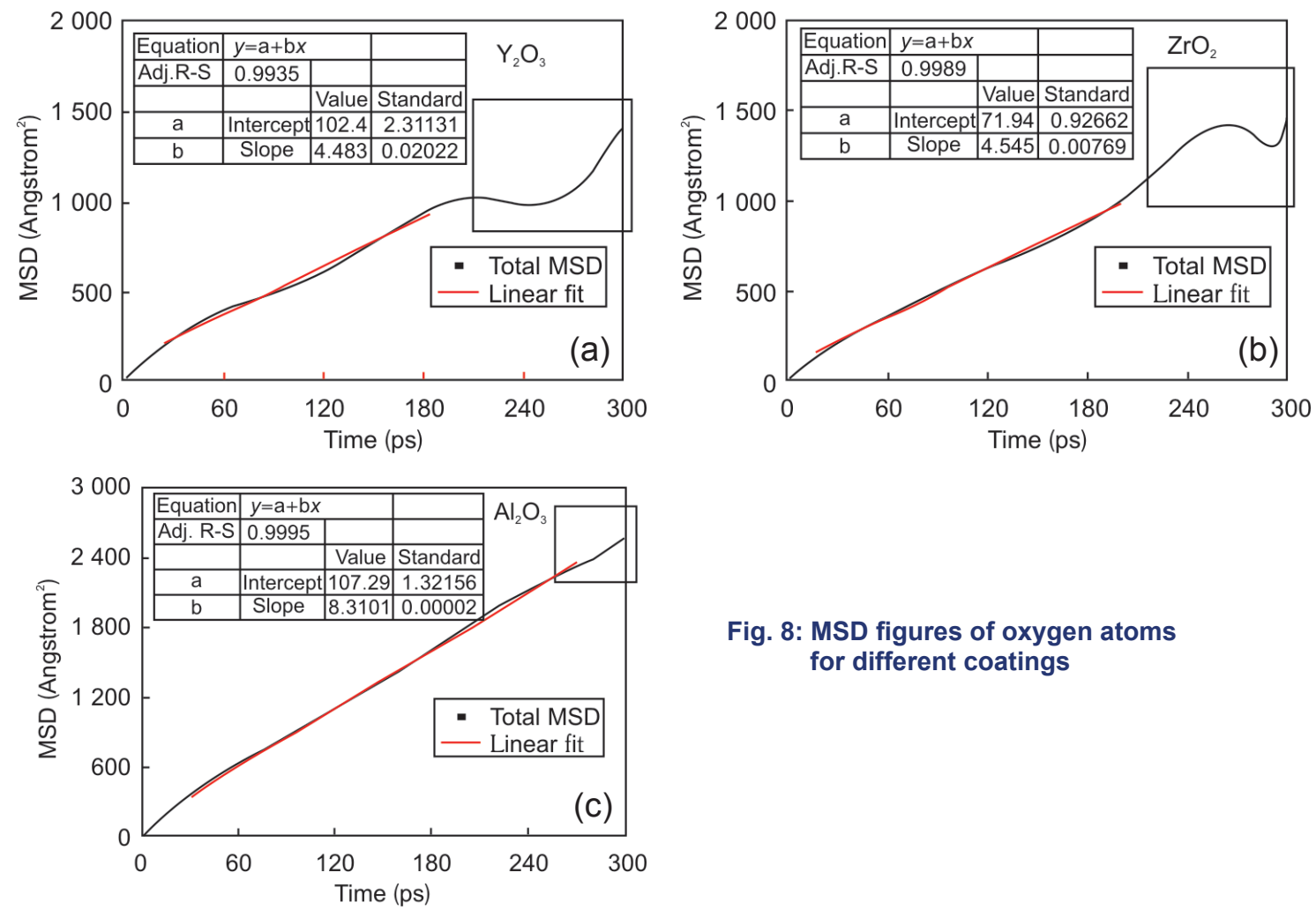

Fig. 8: MSD figures of oxygen atoms for different coatings 
the alumina coating corresponded to the best degree of linear region, which means that the oxygen diffusivity in the alumina coating was relatively preferable. The straight linear area represents diffusion simulation results of each MSD oxygen value in the figures.

The diffusion coefficients $(D)$ were calculated by the MSD. By comparing the anionic position $r_{i}(t)$ with its initial position $r_{0}(t)$, MSD is given by the following equation ${ }^{[21]}$ :

$$
D_{\mathrm{a}}=\frac{1}{6 N_{a}} \lim _{t \rightarrow \infty} \frac{d}{d t} \sum_{i=1}^{N_{a}}\left\langle\left[r_{i}(t)-r_{0}(t)\right]^{2}\right\rangle
$$

where, $r_{i}$ is the position vector of atom $i ; D_{a}$ is the atomic number; $<\left[r_{i}(t)-r_{0}(t)\right]^{2}>$ is ensemble average of the MSD of the gas molecule trajectories; $r_{i}(t)$ and $r_{0}(t)$ are the final and initial position of the center of mass of the gas molecules over the time interval. Equation 2 was applied to each ion type and all molecules in the simulation cell, and $D_{a}$ was calculated between 150 and $250 \mathrm{ps}$ based on the observations that interlayer diffusion can be overestimated if calculated over shorter times.

The slope of the MSD curve that fitted in the linear region was given by the following equation:

$$
D_{a}=b / 6
$$

where, $D_{a}$ is the diffusion coefficient, and $b$ is the parameter corresponding to the thermal vibrations in a crystal.

According to the similar compatible principle, the oxygen diffusion coefficients of different coating materials were similar. So compared with the different oxygen diffusion coefficients in blend system, the compatibility of blends with different coatings can be judged. The tinier the values are, the better the compatibility of blends is ${ }^{[19]}$. The oxygen anion diffusivity was calculated in a time interval of 500 ps and was shown in Fig. 9. The average oxygen anion diffusivity value was estimated to be $0.7472 \times 10^{-4} \mathrm{~cm}^{2} \cdot \mathrm{s}^{-1}$ with $\mathrm{Y}_{2} \mathrm{O}_{3}, 0.7575 \times 10^{-4} \mathrm{~cm}^{2} \cdot \mathrm{s}^{-1}$ with $\mathrm{ZrO}_{2}$ and $1.3850 \times 10^{-4} \mathrm{~cm}^{2} \cdot \mathrm{s}^{-1}$ with $\mathrm{Al}_{2} \mathrm{O}_{3}$, respectively. The negligible differences were observed between the two coatings $\left(\mathrm{Y}_{2} \mathrm{O}_{3}\right.$ and $\mathrm{ZrO}_{2}$ ) from corresponding MSD plots. Thus, the diffusion coefficient of oxygen with $\mathrm{Y}_{2} \mathrm{O}_{3}$ from the truncated plot of long duration simulation was calculated to be larger than the other two coating materials. Therefore, the simulations of a period of 100 ps can be considered reliable enough to calculate the

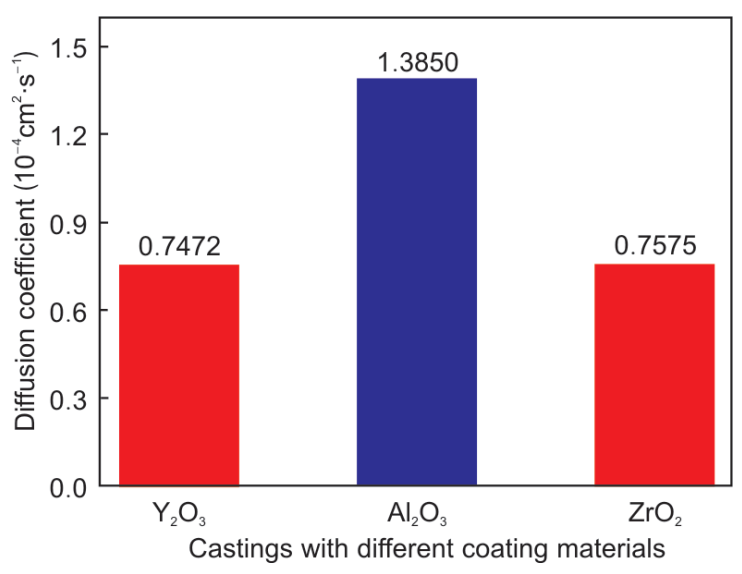

Fig. 9: Diffusion coefficient of oxygen atoms for different coatings diffusivity and predict the trends of diffusion coefficient.

\section{Experiment}

To verify the accuracy of the MD calculated results, Ti-48Al alloys were produced, and its parameters were compared with the simulation results. In this study, aluminum molds (diameter of $6 \mathrm{~mm})$ with different inner coatings $\left(\mathrm{Al}_{2} \mathrm{O}_{3}, \mathrm{Y}_{2} \mathrm{O}_{3}\right.$ and $\left.\mathrm{ZrO}_{2}\right)$ were used during directional solidification. The temperature of the directional solidification furnace was raised to the desired temperature of $1,873 \mathrm{~K}$ for $8 \mathrm{~min}$ and quenched into a molten Ga-In-Sn bath upon reaching the withdrawal distance of $120 \mathrm{~mm}$. The oxygen enrichment of the samples with different coatings is shown in Fig. 10, where the dotted lines represent the interfaces.

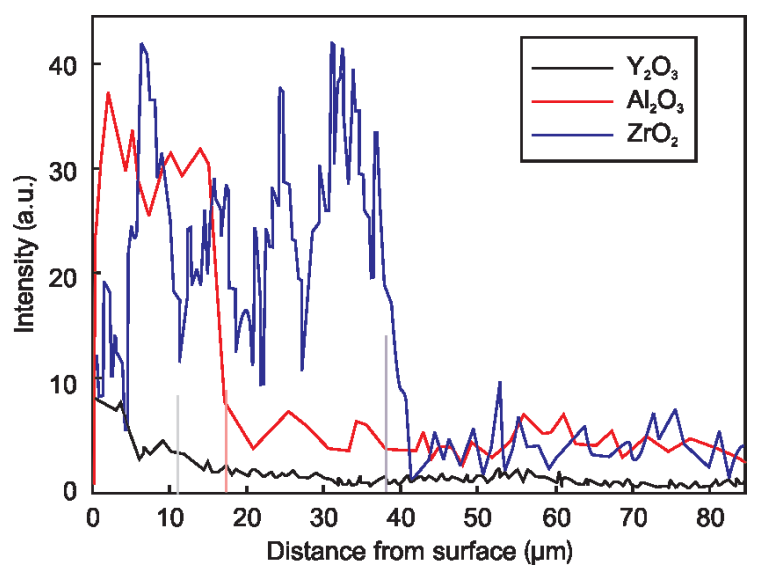

Fig. 10: Oxygen enrichment of samples with different coatings

The results indicate that the oxygen enrichment was the least in the coating region of the sample with $\mathrm{Y}_{2} \mathrm{O}_{3}$, which relatively had the least diffusion distance from the sample surface.

Additionally, severe undulation of oxygen concentration was found in the sample with $\mathrm{ZrO}_{2}$ coating, and it became worse at a distance of $38 \mu \mathrm{m}$ from the surface. In comparison, the oxygen concentration decreased in $\mathrm{Al}_{2} \mathrm{O}_{3}$. However, it also had severe undulation at a distance of $18 \mu \mathrm{m}$ from the surface. The experimental values were well consistent with the simulation data.

\section{Conclusions}

(1) Simulation results demonstrate that the single point energy of $\mathrm{Y}_{2} \mathrm{O}_{3}$ is smaller than that of the other two coating materials. The strength of the intermolecular interaction is in the order of $\mathrm{ZrO}_{2}>\mathrm{Al}_{2} \mathrm{O}_{3}>\mathrm{Y}_{2} \mathrm{O}_{3}$.

(2) The average oxygen anion diffusivity value is estimated to be $0.7472 \times 10^{-4} \mathrm{~cm}^{2} \cdot \mathrm{s}^{-1}$ with $\mathrm{Y}_{2} \mathrm{O}_{3}, 0.7575 \times 10^{-4} \mathrm{~cm}^{2} \cdot \mathrm{s}^{-1}$ with $\mathrm{ZrO}_{2}$ and $1.3850 \times 10^{-4} \mathrm{~cm}^{2} \cdot \mathrm{s}^{-1}$ with $\mathrm{Al}_{2} \mathrm{O}_{3}$, respectively. The diffusion coefficient of oxygen for coating $\mathrm{Y}_{2} \mathrm{O}_{3}$, from the truncated plot of long duration simulation, is larger compared with the other two coating materials.

(3) The oxygen enrichment of Ti-48Al alloy melts with different coatings is obtained in the directional solidification. 
The results indicate that the least oxygen enrichment in the coating region is in the sample with $\mathrm{Y}_{2} \mathrm{O}_{3}$, which relatively has the least diffusion distance from the sample surface. It matches well with simulation data.

\section{References}

[1] Ohtsu N, Ishikawa K, Kobori Y. Hydrogen permeability degradation of Pd-coated $\mathrm{Nb}$-TiNi alloy caused by its interfacial diffusion. Applied Surface Science, 2016, 360: 566-571.

[2] Imayev V, Oleneva T, Imayev R, et al. Microstructure and mechanical properties of low and heavy alloyed $\mathrm{Y}-\mathrm{TiAl}+\mathrm{\alpha}_{2}-\mathrm{Ti}_{3} \mathrm{Al}$ based alloys subjected to different treatments. Intermetallics, 2012, 26: 91-97.

[3] Sui Yanwei, Yang Fang, Li Bangsheng, et al. Physical Simulation Similar Theory and Experiment during Centrifugal Casting Ti Alloy Melts Filling Flow. Rare Metal Materials and Engineering, 2012, 41(8): 1351-1356.

[4] Jo I, Cho S, Kim H, et al. Titanium dioxide coated carbon nanofibers as a promising reinforcement in aluminum matrix composites fabricated by liquid pressing process. Scripta Materialia, 2016, 112: 87-91.

[5] Lapin J, Gabalcová Z, Pelachová T. Effect of $\mathrm{Y}_{2} \mathrm{O}_{3}$ crucible on contamination of directionally solidified intermetallic Ti-46Al8Nb alloy. Intermetallics, 2011, 19(3): 396-403.

[6] Zhang Huarui, Tang Xiaoxia, Zhou Chungen, et al. Comparison of directional solidification of $\mathrm{Y}$-TiAl alloys in conventional $\mathrm{Al}_{2} \mathrm{O}_{3}$ and novel $\mathrm{Y}_{2} \mathrm{O}_{3}$-coated $\mathrm{Al}_{2} \mathrm{O}_{3}$ crucibles. Journal of the European Ceramic Society, 2013, 33(5): 925-934.

[7] Chen Ruirun, Dong Shulin, Guo Jingjie, et al. Investigation of macro/microstructure evolution and mechanical properties of directionally solidified high-Nb TiAl-based alloy. Materials \& Design, 2016, 89: 492-506.

[8] Sui Yanwei, Li Bangsheng, Liu Aihui, et al. Physical Simulation of Infiltration Flow during Centrifugal Casting Titanium Alloy Melts Feeding. Rare Metal Materials and Engineering, 2009, 38(9): 1537-1541.

[9] Luo Wenzhong, Shen Jun, Min Zhixian, et al. Investigation of interfacial reactions between TiAl alloy and crucible materials during directional solidification process. Rare Metal Materials and Engineering, 2009, 38(8): 1441-1445.

[10] Liu Tao, Luo Li, Wang Lei, et al. Influence of thermal stabilization treatment on microstructure evolution of the mushy zone and subsequent directional solidification in Ti43AI-3Si alloy. Materials \& Design, 2016, 97: 392-399.

[11 Chen Ruirun, Zheng Deshuang, Ma Tengfei, et al. Effects of ultrasonic vibration on the microstructure and mechanical properties of high alloying TiAl. Scientific Reports, 2017, 7: 41463.

[12] Sui Yanwei, Feng Kun, Cheng Cheng, et al. Effects of pouring temperature on interfacial reaction between Ti-47.5Al-2.5V-1Cr alloy and mold during centrifugal casting. Journal of Wuhan University of Technology-Mater. Sci. Ed., 2016, 31(5): 11051108.

[13] Cui Ren-jie, Zhang Hua-rui, Tang Xiao-xia, et al. Interactions between $\mathrm{Y}$-TiAl melt and $\mathrm{Y}_{2} \mathrm{O}_{3}$ ceramic material during directional solidification process. Transactions of Nonferrous Metals Society of China, 2011, 21(11): 2415-2420.

[14] Safikhani A, Esmailian M, Tinatiseresht T, et al. High temperature cyclic oxidation behavior of ferritic stainless steel with addition of alloying elements $\mathrm{Nb}$ and $\mathrm{Ti}$ for use in SOFCs interconnect. International Journal of Hydrogen Energy, 2016, 41(14): 6045-6052.

[15] Hou Zhiyuan, Li Yongsheng, Mei Haojie, et al. Lamellar morphology of directional solidified Ti-45Al-6Nb-xW alloys. Rare Metals, 2016, 35(1): 65-69.

[16] Dubbeldam D, Calero S, Ellis D E, et al. RASPA: molecular simulation software for adsorption and diffusion in flexible nanoporous materials. Molecular Simulation, 2016, 42(2): 81101.

[17] Greathouse J A, Cygan R T, Fredrich J T, et al. Molecular Dynamics Simulation of Diffusion and Electrical Conductivity in Montmorillonite Interlayers. The Journal of Physical Chemistry C, 2016, 120(3): 1640-1649.

[18] Marrocchelli D, Merlet C, Salanne M. Molecular Dynamics Simulations of Electrochemical Energy Storage Devices. Physical Multiscale Modeling and Numerical Simulation of Electrochemical Devices for Energy Conversion and Storage. Springer, London, 2016: 61-89.

[19] Sui Yanwei, Feng Kun, Cheng Cheng, et al. Interaction between Ti-48Al Alloy and Coating Materials during the Solidification Process. Rare Metal Materials and Engineering, 2016, 45(12): 3062-3067.

[20] Yusupov M, Neyts E C, Simon P, et al. Reactive molecular dynamics simulations of oxygen species in a liquid water layer of interest for plasma medicine. Journal of Physics D: Applied Physics, 2013, 47(2): 025.

[21] Reuter S, Winter J, Schmidt-Bleker A, et al. Atomic oxygen in a cold argon plasma jet: TALIF spectroscopy in ambient air with modelling and measurements of ambient species diffusion. Plasma Sources Science and Technology, 2012, 21(2): 024.

[22] Kaldenhoff R, Kai L, Uehlein N. Aquaporins and membrane diffusion of $\mathrm{CO}_{2}$ in living organisms. Biochimica et Biophysica Acta (BBA)-General Subjects, 2014, 1840(5): 1592-1595. 